\title{
ORIGINAL ARTICLE \\ Correlates of self-reported physical function in individuals with spinal cord injuries and disorders: does self-efficacy matter?
}

\author{
JN Hill ${ }^{1}$, B Etingen ${ }^{1}$, S Miskevics ${ }^{1}$ and SL LaVela ${ }^{1,2}$
}

\begin{abstract}
Study design: Data were collected via a cross-sectional mailed survey with Veterans with spinal cord injury and disorders (SCI/D). Objectives: To examine self-efficacy in Veterans with SCl/D reporting high versus low perceptions of physical function. Setting: Department of Veterans Affairs (VA) Spinal Cord Injury System of Care-nation-wide, 24 Spinal Cord Injury (SCI) Centers. Methods: The survey provided patient-reported data on demographic and injury characteristics, basic mobility and fine motor function, and perceived self-efficacy. Bivariate comparisons were conducted to compare perceptions of self-efficacy between Veterans with SCI/D reporting perceptions of 'high' versus 'low' basic mobility and fine motor function. A multivariate logistic regression was conducted to identify factors independently associated with high physical function when controlling for covariates.

Results: Response rate $(896 / 1452=61.7 \%)$. Multivariate analysis showed that age (odds ratio $(O R)=0.98,95 \%$ confidence interval $(\mathrm{Cl}): 0.96-1.00, P=0.03$ ), tetraplegia (OR=0.20, 95\% Cl: 0.13-0.32, $P \leqslant 0.0001$ ), diabetes (OR=0.53, 95\% Cl: $0.31-0.91$, $P=0.02)$, depression (OR $=0.62,95 \% \mathrm{Cl}: 0.39-0.98, P=0.04)$ and pressure ulcers $(\mathrm{OR}=0.42,95 \% \mathrm{Cl}: 0.25-0.72, P=0.001)$ were all independently associated with lower odds of high physical function. When controlling for covariates, persons with high self-efficacy were nearly two times more likely to have high physical function $(\mathrm{OR}=1.98,95 \% \mathrm{Cl}: 1.22-3.22, P=0.01)$.

Conclusion: Lower perceptions of basic mobility and fine motor function among individuals with SCI/D were correlated with lower self-efficacy, even when controlling for other covariates. The relationship between physical function and self-efficacy suggests that interventions focused on improving self-efficacy or physical function may also see improvements in the other. Further, studies exploring the impact of interventions on the relationship between self-efficacy and physical function are needed to understand the relationship between the two.
\end{abstract}

Spinal Cord (2017) 55, 1096-1102; doi:10.1038/sc.2017.35; published online 6 June 2017

\section{INTRODUCTION}

There are an estimated 282000 individuals with spinal cord injury (SCI) in the United States (US), ${ }^{1} \sim 42000$ of whom are Veterans. ${ }^{2}$ The Veterans Health Administration (VHA) is the largest integrated health-care system providing care to individuals with spinal cord injuries and disorders (SCI/D), with nearly 29000 Veterans with SCI receiving care through the VA's SCI System of Care, ${ }^{2}$ which includes 24 SCI centers and 134 SCI primary-care clinic teams. ${ }^{3}$

One important area of focus for optimizing overall health and well-being among persons with SCI/D is maximizing function. The term function is a broad construct, which refers to body function and structures, activities and participation that influence and are influenced by aspects of the individual's environment, and personal factors. ${ }^{4}$ The primary aim of rehabilitation following SCI is to equip the patient with skills and strategies to support everyday living, ${ }^{5}$ including a continuous effort to optimize function post rehabilitation and throughout community reintegration efforts over the life course.
One factor relevant in realizing functional goals is self-efficacy; if an individual does not believe that they can achieve the effect they desire, the incentives for performing associated activities are limited. ${ }^{6}$ Self-efficacy is an individual's perception about their capabilities and ability to produce an outcome, and these perceptions mediate their behavior such as impacting course of action, effort given, stress experienced and perseverance when encountering challenges. ${ }^{7}$ Expectations of control, an aspect of self-efficacy, are complicated among individuals with SCI/D by aspects associated with injury such as mobility limitations, which may lead to dependence on paid or informal caregivers. ${ }^{8,9}$ Individuals with SCI who have higher selfesteem and self-efficacy have better mental health, ${ }^{10}$ fewer secondary health conditions ${ }^{11}$ and less overall impairment, ${ }^{12}$ all of which are important aspects of function as classified by the International Classification of Functioning, Disability and Health (ICF). ${ }^{4}$ Further, recent studies have found that self-efficacy acts as a mediator between pain and $\operatorname{mood}^{13}$ and can also be a predictor of outcomes such as social participation, ${ }^{14}$ resilience ${ }^{15}$ and life satisfaction. ${ }^{11}$

${ }^{1}$ Department of Veterans Affairs, Center of Innovation for Complex Chronic Healthcare (CINCCH), Health Services Research and Development, Hines VA Hospital, Hines, IL, USA and ${ }^{2}$ Center for Healthcare Studies, Institute for Public Health and Medicine, General Internal Medicine and Geriatrics, Feinberg School of Medicine, Northwestern University, Chicago, IL, USA

Correspondence: J N Hill, Deparment of Veterans Affairs, Center of Innovation for Complex Chronic Healthcare (CINCCH), Health Services Research and Developmet, Hines VA Hospital, 5000 South 5th Avenue (151H), Hines, IL 60141, USA.

E-mail: Jennifer.Hill3@va.gov

Received 30 September 2016; revised 1 March 2017; accepted 7 March 2017; published online 6 June 2017 
The relationship between self-efficacy and physical activity has been documented in the literature, with evidence suggesting that self-efficacy may be both a determinant and an outcome of physical activity, ${ }^{16,17}$ and an important component of function. In other words, a person's ability to accomplish a physical task may be shaped by what that individual perceives they can do in addition to their actual ability to accomplish that physical task. This relationship has been explored in several interventional studies in individuals with SCI/D related to exercise; these studies demonstrated that interventions supporting physical activity also resulted in improvements to self-efficacy. ${ }^{18,19}$

Assessments of the relationship between self-efficacy and physical function in individuals with SCI/D are limited. In studies of individuals with SCI/D, associations have been found between low self-efficacy and reduced physical function, ${ }^{8}$ whereas higher levels of self-efficacy have been found to be associated with increased physical function and participation. ${ }^{4}$ The purpose of the present study was to examine self-efficacy in Veterans with SCI/D reporting high (vs low) perceptions of elements of their physical function (basic mobility, fine motor function). The a priori hypotheses were that among persons with $\mathrm{SCI} / \mathrm{D}^{1}$, there is a positive association between high physical function and high self-efficacy, and ${ }^{2}$ upon controlling for demographics, injury and health characteristics, high self-efficacy is independently associated with high physical function.

\section{MATERIALS AND METHODS}

\section{Design}

Data were collected via a cross-sectional mailed survey.

\section{Data collection}

From 2014 to 2015, a survey was mailed to a nation-wide random sample of Veterans with SCI/D who had received in-patient and/or outpatient care at a VA facility within the previous year; health-care utilization in the previous year was determined using administrative data available within the VA, and the target sample of 5000 (including 4750 men and 250 women) was selected using SAS PROC SURVEYSELECT, METHOD = PPS (probability proportional to size without replacement). The survey included items to assess demographic and injury characteristics, and a series of established scales to assess basic mobility, fine motor function and self-efficacy (described in detail below). This study was reviewed and approved by the Institutional Review Board at the Edward Hines Jr, VA Hospital to ensure compliance with ethical standards and the study was carried out in an ethical manner.

Variables. Participant characteristics. In an effort to describe the participants, demographic data were collected via patient self-report including the following: sex, age, race/ethnicity, education, marital status and living situation. In addition, the survey was used to gather information on self-reported injuryrelated characteristics including the following: level and completeness of injury and age at which the injury occurred. Participants were also asked to self-report on the presence of select health conditions and symptoms via a questions that asked as follows: In the past 12 months, have you had any of the following healthcare-related complications with a list of responses with responses including diabetes, high blood pressure, high cholesterol, pressure sores, breathing/lung problems, heart-related problems, pain, depression and problems with sleeping. The reported health conditions were also summed to create a cumulative count of the number of health conditions and symptoms reported by each participant. Finally, participants were also asked to rate their perception of their overall general health status (from excellent to poor).

Self-efficacy. To assess aspects of self-efficacy, the University of Washington Self-Efficacy Scale for Spinal Cord Injury was used. ${ }^{7}$ There are two versions of the assessment, a 17-item long form and a 6-item short form, each of which has demonstrated strong psychometric properties, and both the long and short form show evidence of high reliability (Cronbach $\alpha$ of 0.96 and 0.90, respectively), with strong evidence of convergent validity between the forms (correlation coefficients of 0.83 and 0.81 , respectively). ${ }^{7}$ For the purposes of this study, the 6-item short form was used. The response options fall on a 5-point Likert Scale from (1) Not at all to (5) Completely. Higher scores indicate higher perceptions of self-efficacy. The scale covers areas such as the respondent's ability to keep their SCI/D from interfering with things the individual wants to do, dealing with unexpected events, interacting socially and perceived ability to figure out solutions to SCI/D-related issues that come up.

Scoring. Participant's responses to the self-efficacy scale items were summed using the 1 (low) to 5 (high) response option for each item, and then summary/ raw scores were converted to $t$-scores using the conversion tables provided by the scale developers. ${ }^{7}$ Higher scores indicate higher levels of self-efficacy. Since the self-efficacy scale is not normed to population averages, we used a 'one-sample $t$-test' to compare the average self-efficacy score of our sample to a normative average. ${ }^{7}$

Physical function. To measure aspects of physical functioning, this study used two scales from the Spinal Cord Injury-Functional Index; ${ }^{20}$ the Spinal Cord Injury-Functional Index Basic Mobility 11-Item Short Form and Spinal Cord Injury-Functional Index Fine Motor 9-Item Short Form were used to assess collectively physical function. ${ }^{21,22}$ The majority of the questions in the scales had Likert scale response options from (1) Unable to do to (5) Without any difficulty. Some examples of areas covered in the basic mobility subscale include ability to turn the body for pressure reliefs, reach and transfer (e.g., to a toilet or bed). Example areas covered in the fine motor function subscale include ability to make and receive calls on a cellphone, turn the knob on a door and pick up small objects.

Scoring. The basic mobility and fine motor function scales were summed using the 1 (low) to 5 (high) response option for each item and then summary/raw scores were converted to $t$-scores using the conversion tables provided by the scale developers. ${ }^{21}$ To define high (vs low) function, the mean of 50 for both the basic mobility and fine motor function scales was used as the cutoff point; ${ }^{21}$ individuals with above mean (high) scores on both the basic mobility and fine motor function scales were placed in the 'high physical function' group.

ICF. The ICF maps out relationships between the following six components of health: the health condition, body functions and structures, activity, participation, environmental factors and personal factors. ${ }^{20}$ The data collected in this study represent aspects of the ICF in the following ways: body structure and function (e.g., level of injury, health status and certain health conditions), personal factors (e.g. age, age at injury and self-efficacy), environment (living situation) and physical function (basic mobility, fine motor function). ${ }^{4}$ Aspects of personal factors within the ICF such as self-effiacacy and self-esteem have been tied to physical participation and functional status; ${ }^{20}$ therefore, self-efficacy was incorporated as a personal factor in the current study.

Data analysis. Bivariate comparisons (i.e., Student's $t$-tests, $\chi^{2}$ tests) were conducted to assess demographics, injury characteristics, health-related factors and perceptions of self-efficacy by individuals with high vs low physical function. Bonferroni corrections were added to adjust the $P$-value for multiple comparisons.

A multivariate logistic regression was conducted to identify factors independently associated with high physical function when controlling for covariates. Selections for covariates to include in the model were based on the following criteria: significant bivariate associations, associations with physical function identified in the literature and a priori hypotheses.

Persons with complete data on all covariates were included in the final model, and model fit was assessed using log-likelihood testing. We examined differences in demographics, injury characteristics and select health conditions for respondents who were included in the final regression model vs those who did not make it into the model due to incomplete data.

The final model included the following covariates: age (continuous); tetraplegia (reference group: paraplegia); high self-efficacy (reference group: low self-efficacy); excellent/very good/good self-reported health status (reference group: fair/poor); diabetes (reference group: no diabetes), depression (reference group: no depression), pressure ulcers (reference group: no pressure ulcers) and heart disease (reference group: no heart disease). 
Table 1 Participant characteristics by low vs high physical function

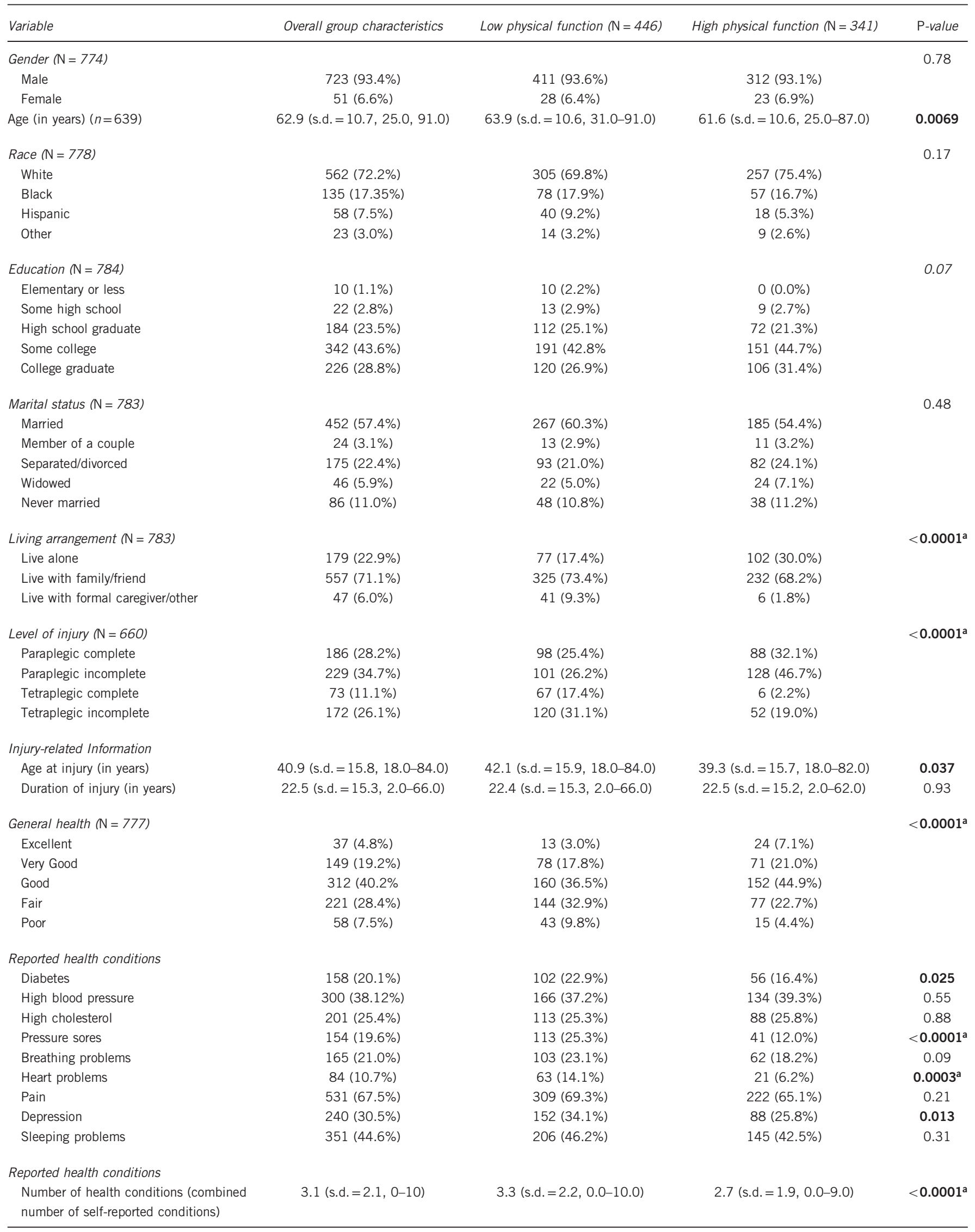

aSignificant after Bonferonni correction (modified $P$-value $=0.0025$ ). Bold values indicates significance. Italic values indicates approaching significance. 
An $\alpha$ level of 0.05 was used to determine statistical significance. Statistical analyses were performed with SAS 9.2 (SAS Institute Inc., Cary, NC, USA).

\section{RESULTS}

Surveys were sent to 1786 Veterans with SCI/D; of those surveys, 271 were undeliverable and were returned by the post office, 50 individuals had passed away since the list of potential participants was generated, and 13 individuals returned the survey blank because it was not applicable to them. Therefore, the total number of potential respondents was 1452, of whom 896 individuals with SCI/D (61.7\% response rate) responded to the survey.

\section{Demographics, injury characteristics and health-related factors}

For respondents used in the comparison groups $(N=787)$, respondents in the high (vs low) physical function group reported a lesser number of health conditions and symptoms overall (2.7 vs 3.1, $<0.0001$ ) (Table 1). When looking at certain health conditions/ symptoms, a lesser proportion of respondents in the high (vs low) physical function group reported prior-year: pressure sores $(12.0 \%$ vs $25.3 \%, P \leqslant 0.0001)$ and heart problems $(6.2 \%$ vs $14.1 \%, P \leqslant 0.0001)$.

\section{Physical function}

Individuals with SCI/D in this study had an overall mean basic mobility score of 49.1 (s.d. =7.4, $\min 30.2$, $\max 63.9$ ) (Figure 1). For fine motor function, the overall mean score was 50.4 (s.d.=7.6, min 31.4, max 62.6) (Figure 2).

Slightly over half $(53.1 \%)$ of the respondents reported above mean ( 50.0 or greater) 'high' basic mobility, and $53.5 \%$ reported above mean (50.0 or greater) 'high' fine motor function. Just under half $(43.3 \%)$ of respondents reported above mean scores on both the basic mobility and fine motor function scales and were classified as having reported 'high physical function'.

\section{Self-efficacy}

Respondents in the study had an overall mean self-efficacy score of 44.7 (s.d.=9.7, range: 20.0-68.9) (Table 2). Our respondents reported lower average self-efficacy compared with the normative mean (49.9), $t(837)=-17.16, P<0.0001$. Overall, respondents reported the highest self-efficacy $(\mathrm{M}=3.26$ (s.d. $=1.1$, range: $1.0-5.0))$ on question 5 : 'How confident are you that you can bounce back from frustration, discouragement, or disappointment that SCI may cause you?' The lowest reported self-efficacy score, $M=2.7$ (s.d. $=1.1$, range: $1.0-5.0$ ), was reported on question 2: 'You can keep your SCI from interfering with your ability to deal with unexpected events?'

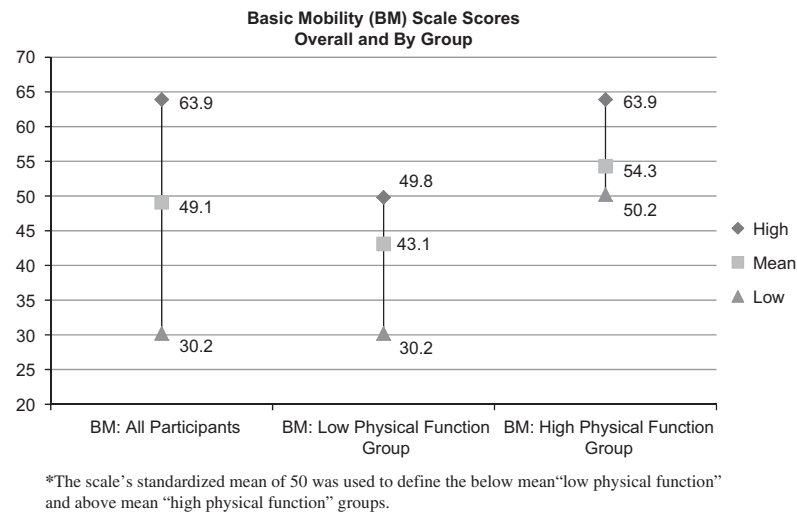

Figure 1 Perceptions of basic mobility in high vs low physical function groups.

\section{Self-efficacy and physical function}

Veterans with high (vs low) basic mobility reported higher perceptions of self-efficacy on average ( 46.2 vs $41.7, P<0.0001)$ (Table 2). Similarly, Veterans with high (vs low) fine motor function reported higher average perceptions of self-efficacy ( 45.9 vs $41.8, P<0.0001$ ). When the physical function measures of basic mobility and fine motor function were considered collectively, Veterans with high (vs low) physical function reported higher mean perceptions of self-efficacy (46.6 vs $42.2, P<0.0001$ ).

\section{Multivariate logistic regression}

Compared with those who were not included in the final regression model due to incomplete data, individuals who were included in the model were younger, on average (62.4 vs 64.7 years old, $P=0.02)$ and had sustained their SCI at a younger age (39.6 vs 46.1 years old, $P<0.0001)$; a greater proportion reported having completed some college $(47.6 \%$ vs $37.3 \%, P=0.01)$ and having experienced prior-year pressure sores $(21.8 \%$ vs $16.1 \%, P=0.05)$ and pain $(70.5 \%$ vs $62.6 \%$, $P=0.02)$. There were no other differences in demographics, injury characteristics or health conditions among respondents who were included in the final regression model compared with those who were not.

The results of the multivariate logistic regression (Table 3) model to assess factors independently associated with high physical function and the analyses included 482 individuals. Data indicated that younger age (odds ratio $(\mathrm{OR})=0.98,95 \%$ confidence interval $(\mathrm{CI})$ : 0.96-1.00, $P=0.03)$, tetraplegia $(\mathrm{OR}=0.20,95 \%$ CI: $0.13-0.32, P=<0.0001)$ and presence of diabetes $(\mathrm{OR}=0.53,95 \% \mathrm{CI}: 0.31-0.91, P=0.02)$, depression $(\mathrm{OR}=0.62,95 \% \mathrm{CI}: 0.39-0.98, P=0.04)$ and pressure ulcers $(\mathrm{OR}=0.42,95 \% \mathrm{CI}: 0.25-0.72, P=0.001)$ were all independently associated with lower odds of high physical function. When controlling for covariates, persons with high self-efficacy $(\mathrm{OR}=1.98$, $95 \%$ CI: $1.22-3.22, P=0.01)$ were nearly two times more likely to have high physical function.

\section{DISCUSSION}

This study presents results that explored the associations of several factors on perceived functioning in persons with SCI/D. The present study found that living alone and having a paraplegic injury were associated with high perceptions of physical function. This study also found that having a higher number of comorbid health conditions and symptoms, and having certain conditions (diabetes, depression, pressure sores and heart problems), was associated with lower perceptions of physical function. While these relationships may not be surprising, they indicate that greater attention to promoting positive perceptions of functional ability may be needed, especially in individuals with SCI/D who are at increased risk for developing or who already have secondary complications and comorbid conditions. Of note, it is possible that having health complications negatively impacts physical function but also that decreased physical function may lead to the development of health complications; however, the direction of this relationship cannot be determined from the data in the present study.

\section{Relationship between self-efficacy and physical function}

Self-efficacy has been documented as an important factor for persons with SCI/D, having into a variety of SCI rehabilitation outcomes including physical function. ${ }^{8,11,14,15}$ Self-efficacy has been shown to influence perceptions of well-being and physical function. ${ }^{17}$ The current study offers additional supporting evidence of this relationship, but in a new population, supporting the concept that 
the relationship between self-efficacy and physical function might be generalizable to other populations. In this study, differences in self-efficacy existed between individuals with SCI/D reporting high vs low perceptions of physical function.

Further, the data presented in the current study suggest that even while controlling for other covariates including aspects of body structure and function (such as the level of injury, health status and certain health conditions/symptoms), personal factors (such as age, age at injury and self-efficacy) and environment (e.g., living situation), self-efficacy was an important correlate, in that individuals with high self-efficacy (personal factor) were nearly two times more likely to report high physical function (activities and participation). A review of the literature found that aspects related to personal factors within the ICF such as self-efficacy and self-esteem are critical to participation in various ways including physical participation and functional status. ${ }^{23}$

Similar studies have found that low self-efficacy is associated with reduced quality of life beyond the effect of the physical impairments associated with $\mathrm{SCI}^{8,9}$ and that self-efficacy and belief in the ability to cope with disability is protective against the development of depression and anxiety among individuals with SCI. ${ }^{8}$ In a study of individuals with SCI, both self-efficacy and self-esteem were examined as

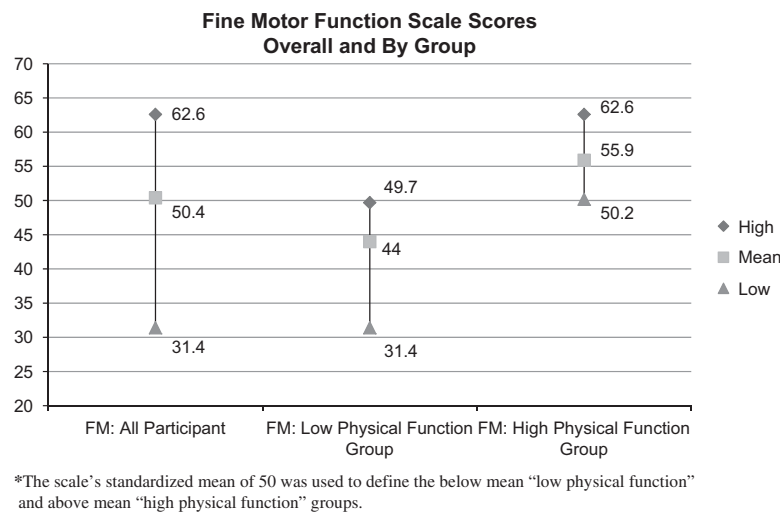

Figure 2 Perceptions of fine motor function in high vs low physical function groups. predictors of participation and both were strongly related to the level of participation (stronger than symptoms of anxiety, depressive symptoms, pain, health conditions, social support, coping styles or a sense of coherence). ${ }^{4}$ While data from the present study cannot directly ascertain whether self-reported physical function influences self-efficacy or vice versa, given the importance of retaining physical function after SCI and the influence of self-efficacy on elements of physical function, interventions to promote self-efficacy to support maintenance and improvements in physical function in individuals with SCI/D are warranted.

Examples of existing intervention studies suggest that self-efficacy and physical function in individuals with SCI/D share strong ties and demonstrate a broad range of possible interventions that could support these improvements from less invasive (e.g., massage therapy and peer mentoring) to more invasive (e.g., surgery) intervention strategies. ${ }^{5,24,25}$ For example, in a study of individuals

Table 3 Multivariate logistic regression: factors associated with high physical function among Veterans with $\mathrm{SCl} / \mathrm{D}$

\begin{tabular}{lccc}
\hline Covariates $^{\mathrm{a}}$ & OR & $95 \% \mathrm{Cl}$ & P-value \\
\hline Age (continuous) & 0.98 & $0.96-1.00$ & 0.03 \\
Living situation (lives alone) & 1.57 & $0.95-2.58$ & 0.08 \\
Level of injury (tetraplegia) & 0.20 & $0.13-0.32$ & $<0.0001$ \\
Age at injury (continuous) & 0.99 & $0.98-1.01$ & 0.30 \\
Self-efficacy (high self-efficacy) & 1.98 & $1.22-3.22$ & 0.01 \\
Self-reported health status (excellent/very & 0.88 & $0.53-1.48$ & 0.63 \\
good/good) & & & \\
Diabetes (no) & 0.53 & $0.31-0.91$ & 0.02 \\
Depression (no) & 0.62 & $0.39-0.98$ & 0.04 \\
Pressure ulcers (no) & 0.42 & $0.25-0.72$ & 0.001 \\
Heart disease (no) & 0.58 & $0.28-1.22$ & 0.15 \\
\hline
\end{tabular}

Age (continuous). Living situation (lives alone); reference group: lives with family/friend, lives with formal caregiver/other. Level of injury (tetraplegia); reference group: tetraplegia. Age at injury (continuous). Self-efficacy (high self-efficacy); reference group: low self-efficacy. Selfreported health status (excellent/very good/good); reference group: fair, poor. Diabetes (yes); reference group: no. Depression (yes); reference group: no. Pressure ulcers (yes); reference group: no. Heart disease (yes): reference group: no.

${ }^{a}$ Covariates and reference groups. Bold values indicates significance. Italic values indicates approaching significance.

Table 2 Self-efficacy by high vs low physical function (basic mobility and fine motor function) in veterans with SCI/D

\begin{tabular}{|c|c|c|c|c|}
\hline Variable & $\begin{array}{l}\text { Overall sample } \\
\qquad(\mathrm{N}=773)\end{array}$ & $\begin{array}{l}\text { Low physical function } \\
\qquad(\mathrm{N}=434)\end{array}$ & $\begin{array}{l}\text { High physical function } \\
\qquad(\mathrm{N}=339)\end{array}$ & P-value \\
\hline Overall self-efficacy score mean (s.d., range) & $\begin{array}{c}44.7 \\
\text { (s.d. }=9.7,20.0-68.9)\end{array}$ & $\begin{array}{c}42.2 \\
(\text { s.d. }=9.6,20.0-68.9)\end{array}$ & $\begin{array}{c}46.6 \\
(\mathrm{~s} . \mathrm{d} .=9.2,23.8-68.9)\end{array}$ & $<0.0001^{\mathrm{a}}$ \\
\hline \multicolumn{5}{|l|}{ Individual items (range: $1.0-5.0)^{\mathrm{b}}$ : mean (s.d.) } \\
\hline \multicolumn{5}{|l|}{ How confident are you that } \\
\hline $\begin{array}{l}\text { Q1. You can keep the physical discomfort of your } \mathrm{SCl} \text { from interfering with things } \\
\text { you want to do? }(N=773)\end{array}$ & 2.8 (s.d. $=1.2)$ & 2.6 (s.d. $=1.2$ ) & 3.0 (s.d. $=1.1$ ) & $<0.0001^{a}$ \\
\hline $\begin{array}{l}\text { Q2. You can keep your } \mathrm{SCI} \text { from interfering with your ability to deal with } \\
\text { unexpected events? }(N=773)\end{array}$ & 2.7 (s.d. = 1.1) & 2.6 (s.d. = 1.2) & 3.0 (s.d. = 1.1) & $<0.0001^{a}$ \\
\hline $\begin{array}{l}\text { Q3. You can keep your SCI from interfering with your ability to interact socially? } \\
(N=770)\end{array}$ & 2.9 (s.d. = 1.2) & 2.7 (s.d. = 1.2) & 3.2 (s.d. = 1.2) & $<0.0001^{a}$ \\
\hline Q4. You can keep your $\mathrm{SCl}$ from being the center of your life? $(N=770)$ & 2.9 (s.d. = 1.2) & 2.7 (s.d. = 1.2) & 3.2 (s.d. = 1.2) & $<0.0001^{\mathrm{a}}$ \\
\hline $\begin{array}{l}\text { Q5. You can bounce back from frustration, discouragement, or disappointment } \\
\text { that } \mathrm{SCl} \text { may cause you? }(N=770)\end{array}$ & 3.26 (s.d. = 1.1) & 3.0 (s.d. 1.1) & 3.5 (s.d. = 1.1) & $<0.0001^{a}$ \\
\hline $\begin{array}{l}\text { Q6. You can figure out effective solution to SCl-related issues that come up? } \\
(N=769)\end{array}$ & 3.23 (s.d. = 1.1) & 3.0 (s.d. = 1.1) & 3.5 (s.d. = 1.0) & $<0.0001^{a}$ \\
\hline
\end{tabular}

asignificant after Bonferroni correction (modified $P$-value $=0.007$ ).

bRange of responses for all individual items was 1.0-5.0. Bold values indicates significance. 
with multiple sclerosis, an 8-week massage therapy intervention focused on improving self-efficacy through therapeutic massage resulted in improvements of self-efficacy within 4 weeks of the start of intervention and sustained 4 weeks after the intervention ended. ${ }^{25}$ The majority of participants saw improvements in self-efficacy, and these improvements were higher among people with less education. ${ }^{24}$ Finally, in another study of individuals with tetraplegia who had received reconstructive hand surgery to regain hand function, researchers found that the resulting increase in self-efficacy related to hand control parlayed into additional functional improvements and further increases in self-efficacy. ${ }^{5}$

\section{Implications}

Previous studies have suggested that self-efficacy and physical activity (related to physical function) are modifiable factors that influence perceptions of physical ability as well as functional limitations associated with chronic disease and aging. ${ }^{5,17,25}$ Self-efficacy has also been linked to other aspects of health and well-being. Negative perceptions of control and self-efficacy are associated with decreased optimism. ${ }^{26}$ In a study of individuals with SCI/D, individuals with low self-efficacy had significantly elevated depressive mood and elevated anxiety compared with those with high self-efficacy. ${ }^{8}$ The broader impact of self-efficacy was demonstrated in a recent systematic review, which found that it was one of the four concepts that were consistently linked to quality of life for individuals with SCI/D. ${ }^{27,28}$ Further, a recent study of community-dwelling manual wheelchair users over the age of 50 years concluded that self-efficacy both directly and indirectly impacts participation frequency, and that interventions addressing low self-efficacy are warranted. ${ }^{29}$

Self-efficacy can be a key factor in fostering development of daily life skills as well as participation in society and self-reflection. ${ }^{5}$ Enhancing self-efficacy may be effective in supporting daily activities such as ambulating, reaching and lifting and generally interacting with one's physical environment. ${ }^{16} \mathrm{SCI}$ rehabilitation should include components that heighten self-efficacy by facilitating feelings of control, which may be influenced by supporting improvements in function and promoting independence. ${ }^{28}$ Enhancing self-efficacy through education and rehabilitation programs and measurement of self-efficacy as a result of these activities have the potential to improve outcomes for individuals with SCI/D. Based on the present study's findings, incorporating patient preferences into the selection and design of interventions targeting improvements in mobility and function is recommended to ensure that patient-centric needs are being met.

\section{Study limitations}

The findings of this study should be interpreted while considering a number of limitations. First, the cross-sectional nature of the study is observational and descriptive; therefore, causality cannot be inferred. Second, the data were collected via a self-report survey, and therefore certain biases such as participation/non-participation bias, recall and comprehension problems may be present. Third, the study included primarily male patients within the VA health-care system, where female representation is typically low; therefore, generalization of the results to female patients may not be appropriate. Fourth, the response rate of $61.7 \%$ may be considered low, but is similar to response rates of other mailed surveys to Veterans reporting $49-67 \%$ response rates, ${ }^{30,31}$ and is higher than a previously reported mailed survey of Veterans with SCI/D, which reported a $38 \%$ response rate. ${ }^{32}$ Fifth, there was a drop in sample size between total number of respondents and those included in the logistic regress; only those individuals who had complete data were included in the logistic regression, which may have implications related to the study findings. Sixth, the measures used in this analysis are not fully representative of components of the ICF; for example, the measure of social support (living situation) does not fully represent the scope of environmental factors as described by the ICF. ${ }^{4}$ Finally, the data reported on Veterans with SCI/D may not be fully representative of the broader population of individuals with $\mathrm{SCI} / \mathrm{D}$, although as reported, the findings of this study are comparable to other studies of individuals with SCI using the same measures. ${ }^{7,20,22}$

\section{CONCLUSION}

In the context of chronic incurable disease/disability, an individual can achieve a healthy outlook ${ }^{25}$ and individuals need to be empowered and compelled to take steps to support their own optimal health and wellbeing. ${ }^{33}$ Lower perceptions of physical function (i.e., mobility and fine motor function) among individuals with SCI/D are associated with lower self-efficacy. Interventions focused on promoting and empowering physical function, including basic mobility and fine motor skills (e.g., supporting regular physical activity appropriate for the patient) may both improve elements of functionality, such as basic mobility and fine motor function, and bolster self-efficacy.

\section{DATA ARCHIVING}

There were no data to deposit.

\section{CONFLICT OF INTEREST}

The authors declare no conflict of interest.

\section{ACKNOWLEDGEMENTS}

The data presented in this manuscript have not been published and will not be submitted elsewhere for publication while being considered in this journal. Data on this study were accepted as a platform presentation and will be presented, in part, at the 2016 Paralyzed Veterans of America 6th Annual Summit and Expo on 1 September 2016 in Orlando, Florida. This study was supported by the Department of Veterans Affairs, Health Services Research and Development, Quality Enhancement Research Initiative (RRP 13-248, PI: LaVela). The views expressed in this presentation are those of the authors and do not necessarily reflect the position or policy of the Department of Veterans Affairs or the United States government.

1 National Spinal Cord Injury Statistical Center. Spinal Cord Injury (SCI) Facts and Figures at a Glance. University of Alabama-Birmingham: Birmingham, AL, USA, 2016.

2 United States Department of Veterans Affairs. Spinal Cord Injury Fact Sheet [Internet]. Washington (DC): U.S. Department of Veterans Affairs; 2009 Jan [cited 2017 Feb 6]. Available at: http://www.va.gov/opa/publications/factsheets/fs_spinal_cord_injury.pdf.

3 United States Department of Veterans Affairs. Spinal Cord Injuries System of Care [Internet]. Washington (DC): U.S. Department of Veterans Affairs; 2017 Jan [cited 2017 Feb 6]. Available at: http://www.sci.va.gov/NAs_SCID_System_of_Care.asp.

4 Geyh S, Nick E, Stirnimann D, Ehrat S, Michel F, Peter C et al. Self-efficacy and self-esteem as predictors of participation in spinal cord injury an ICF-based study. Spinal Cord 2012; 50: 699-706.

5 Wangdell J, Carlsson G, Friden J. Enhanced independence: experiences after regaining grip function in people with tetraplegia. Disabil Rehabil 2013; 35: 1968-1974.

6 Bandura A. Self-efficacy. Corsini Encyclopedia of Psychology, 4th edn. Wiley: Hoboken, NJ, USA, 2010.

7 Amtmann D, Bamer AM, Cook KF, Askew RL, Noonan VK, Brockway JA. University of Washington self-efficacy scale: a new self-efficacy scale for people with disabilities. Arch Phys Med Rehabil 2012; 93: 1757-1765.

8 Craig A, Wijesuriya N, Tran Y. The influence of self-efficacy on mood states in people with spinal cord injury. ISRN Rehabil 2013; 2013: 1-6.

9 Craig A, Tran Y, Middleton J. Psychological morbidity and spinal cord injury: a systematic review. Spinal Cord 2009; 47: 108-114.

10 van Leeuwen C, Edelaar-Peeters Y, Peter C, Stiggelbout AM, Post MW. Psychological factors and mental health in persons with spinal cord injury: an exploration of change or stability. J Rehabil Med 2015; 47: 531-537.

11 Cijsouw A, Adriaansen JJE, Tepper M, Dijksta CA, van Linden S, van der Woude LHV et al. Associations between disability-management self-efficacy, participation and life 
satisfaction in people with long-standing spinal cord injury. Spinal Cord 2017; 55: 47-51.

12 Peter C, Muller R, Cieza A, Geyh S. Psychological resources in spinal cord injury: a systematic literature review. Spinal Cord 2012; 50: 188-201.

13 Craig A, Tran Y, Siddall P, Wijesuriya N, Lovas J, Bartrop R et al. Developing a model of associations between chronic pain, depressive mood, chronic fatigue and self-efficacy in people with spinal cord injury. J Pain 2013; 14: 911-920.

14 Craig A, Nicholson Perry K, Guest R, Tran Y, Middleton J. Adjustment following chronic spinal cord injury: determining factors that contribute to social participation. $\mathrm{Br} \mathrm{J}$ Health Psychol 2015; 20: 807-823.

15 Guest R, Craig A, Tran Y, Middleton J. Factors predicting resilience in people with spinal cord injury during transition from inpatient rehabilitation to the community. Spinal Cord 2015; 53: 682-686.

16 McAuley E, Szabo A, Gothe N, Olson EA. Self-efficacy: Implications for physical activity, function, and functional limitations in older adults. Am J Lifestyle Med 2011; 5: 361-369.

17 McAuley E, Morris KS, Doerksen SE, Motl RW, Liang H, White SM et al. Effects of change in physical activity on physical function limitations in older women: mediating roles of physical function performance and self-efficacy. J Am Geriatr Soc 2007; 55: 1967-1973.

18 Ginis KAM, Tomasone JR, Latimer-Cheung AE, Arbour-Nicitopoulos KP, Bassett-Gunter RL, Wolfe DL. Developing physical activity interventions for adults with spinal cord injury. Part 1: a comparison of social cognitions across actors, intenders, and nonintenders. Rehabil Psychol 2013; 58: 299-306.

19 Nooijen CF, Stam HJ, Sluis T, Valent L, Twisk J, van den Berg-Emons RJ. A behavioral intervention promoting physical activity in people with subacute spinal cord injury: Secondary effects on health, social participation and quality of life. Clin Rehabil 2017; 31: 772-780.

20 Jette AM, Tulsky DS, Ni P, Kisala PA, Slavin MD, Dijkers MP et al. Development and initial evaluation of the spinal cord injury-functional index. Arch Phys Med Rehabil 2012; 93: 1733-1750.

21 Tulsky DS, Jette AM, Kisala PA, Kalpakjian C, Dijkers MP, Whiteneck G et al. Spinal cord injury-functional index: item banks to measure physical functioning in individuals with spinal cord injury. Arch Phys Med Rehabil 2012; 93: 1722-1732.
22 Heinemann AW, Dijkers MP, Ni P, Tulsky DS, Jette A. Measurement properties of the spinal cord injury-functional index (SCI-FI) short forms. Arch Phys Med Rehabil 2014; 95: 1289-1297.

23 Geyh S, Peter C, Muller R, Bickenbach JE, Kostanjsek N, Ustun BT et al. The personal factors of the International Classification of Functioning, Disability and Health in the literatureGÇôa systematic review and content analysis. Disabil Rehabil 2011; 33: 1089-1102.

24 Ljungberg I, Kroll T, Libin A, Gordon S. Using peer mentoring for people with spinal cord injury to enhance self-efficacy beliefs and prevent medical complications. J Clin Nurs 2011; 20: 351-358.

25 Finch $\mathrm{P}$, Bessonnette $\mathrm{S}$. A pragmatic investigation into the effects of massage therapy on the self efficacy of multiple sclerosis clients. J Bodyw Mov Ther 2014; 18: 11-16.

26 Sinnakaruppan I, Macdonald K, McCafferty A, Mattison P. An exploration of the relationship between perception of control, physical disability, optimism, self-efficacy and hopelessness in multiple sclerosis. Int J Rehabil Res 2010; 33: 26-33.

27 van Leeuwen CMC, Kraaijeveld S, Lindeman E, Post MWM. Associations between psychological factors and quality of life ratings in persons with spinal cord injury: a systematic review. Spinal Cord 2012; 50: 174-187.

28 Middleton J, Tran Y, Craig A. Relationship between quality of life and self-efficacy in persons with spinal cord injuries. Arch Phys Med Rehabil 2007; 88: 1643-1648.

29 Sakakibara BM, Miller WC, Routhier F, Backman CL, Eng JJ. Association between self-efficacy and participation in community-dwelling manual wheelchair users aged 50 years or older. Phys Ther 2014; 94: 664-674.

30 Street AE, Gradus JL, Giasson HL, Vogt D, Resick PA. Gender differences among veterans deployed in support of the wars in Afghanistan and Iraq. J Gen Intern Med 2013; 28: S556-S562.

31 Tsai J, Rosenheck RA. Use of the internet and an online personal health record system by US veterans: comparison of Veterans Affairs mental health service users and other veterans nationally. J Am Med Inform Assoc 2012; 9: 1089-1094.

32 Hogan TP, Hill JN, Locatelli SM, Weaver FM, Thomas FP, Nazi KM et al. Health information seeking and technology use among veterans with spinal cord injuries and disorders. PM R 2016; 8: 123-130.

33 Mattila E, Korhonen I, Salminen JH, Ahtinen A, Koskinen E, Syrely A et al. Empowering citizens for well-being and chronic disease management with wellness diary. IEEE Trans Inform Technol Biomed 2010; 14: 456-463. 